\title{
AVALIAÇÃO DA FUNÇÃO RENAL EM PACIENTES PORTADORES DE RIM ÚNICO
}

\author{
EVALUATION OF RENAL FUNCTION IN SOLITARY KIDNEYS PATIENTS
}

Felipe de Paula Saboia ${ }^{1}$, Mohamad Abdul Majid Chams ${ }^{1}$, Marcelo Mazza do Nascimento ${ }^{1}$

\section{RESUMO}

Introdução: Tem sido descrito na literatura que pacientes portadores de rim único podem desenvolver perda da função renal tornando-se portadores de doença renal crônica (DRC). Objetivo: Avaliar a função renal em pacientes nefrectomizados e acompanhados no HC-UFPR. Métodos: Estudo retrospectivo, analítico e descritivo. Os pacientes foram selecionados no sistema de informática por meio do CID-Z52.4 (doador renal) e CID-Z90.5 (ausência adquirida do rim). Foram selecionados 28 pacientes, os quais foram acompanhados no período entre 1984 e 2016 . Foram coletados dados clínicos e laboratoriais dos prontuários ao longo do acompanhamento. Foi avaliada a função renal por meio da estimativa da Taxa de Filtração Glomerular (TFG), calculada usando a fórmula CKD-EPI (Chronic Kidney Disease - Epidemiology collaboration equation) e pela quantificação de proteinúria. Resultados: A população estudada foi majoritariamente do sexo feminino (67\%) e possuía média ( \pm desvio padrão) de idade de $56 \pm 12$ anos. Deste total, $75 \%$ eram doadores renais, $21 \%$ foram nefrectomizados por complicações de nefrolitíase e $4 \%$ por estenose de junção uretero-piélica. Desses, $57 \%$ dos pacientes eram hipertensos e $11 \%$, diabéticos. O tempo de acompanhamento médio foi de 14,64 $\pm 9,02$ anos. Observou-se uma queda insignificante na TFG estimada $\left(79,6 \pm 21,95\right.$ versus $\left.69,3 \pm 20,52 \mathrm{ml} / \mathrm{min} / 1,73 \mathrm{~m}^{2}, \mathrm{p}=0,14\right)$ no acompanhamento. Entretanto, observou-se aumento prevalência de proteinúria, inicialmente observada em 10,71\%, para 39,29\% ao final do acompanhamento $(p<0,05)$. Conclusão: Apesar de não se verificar uma queda significativa da TFGe, verificou-se o aumento da prevalência de proteinúria, apontando a possibilidade, destes indivíduos, desenvolverem doença renal crônica a longo prazo.

Descritores: Doador renal. Nefrectomia. Rim único. Proteinúria. Função Renal.

\section{ABSTRACT}

Introduction: It has been reported in the literature that patients with solitary kidney could develop chronic kidney disease over the time. Objective: Evaluation of renal function in solitary kidney patients of the HC-UFPR nephrology service. Methods: A retrospective, analytical and descriptive study. Patients were initially selected by searching for the ICD codes Z52.4 (kidney donor) and Z90.5 (acquired absence of the kidney) in the hospital's patient database. Twenty-eight patients, followed in the period between 1984 and 2016, were included in the study. Clinical data and laboratory results were extracted from the patients' medical records. Renal function was evaluated by the glomerular filtration rate (GFR), estimated using the CKD-EPI formula (Chronic Kidney Disease - Epidemiology Collaboration Equation) and by the quantification of proteinuria. Results: The studied sample was predominantly female (67\%) and had a mean age of $56 \pm 12$ years. Seventy-five percent of the studied population were kidney donors, $21 \%$ have been nephrectomized following complications of nephrolithiasis and $4 \%$, as a consequence to stenosis of the ureteropelvic junction. Fifty-seven percent of the patients were hypertensive and $11 \%$, diabetic. The mean follow-up time was $14.64 \pm 9.02$ years. There was an insignificant decrease in estimated GFR $(79.6 \pm 21.95$ versus $20.52 \pm 69.3 \mathrm{ml} / \mathrm{min} / 1.73 \mathrm{~m} 2, \mathrm{p}=0.14)$. However, the prevalence of proteinuria increased from $10.71 \%$ to $39.29 \%(p$ $<0.05)$. Conclusions: Although there was no significant decrease in the GFR, an increased prevalence of proteinuria during follow-up was observed, which points towards the possibility of these individuals developing chronic kidney disease over time.

Keywords: Kidney Donor. Nephrectomy. Solitary Kidney. Proteinuria. Renal Function.

1 - Hospital de Clínicas da Universidade Federal do Paraná, Curitiba, Brasil.

Contato do Autor / Mail to:

Marcelo Mazza do Nascimento

Rua General Carneiro, 181 - Alto da Glória, Curitiba, Paraná, Brasil - CEP 80.060-900 


\section{REVISTA MÉDICA DA UFPR}

\section{INTRODUÇÃO}

O transplante renal tornou-se uma alternativa à terapia dialítica em pacientes renais crônicos, melhorando a qualidade de vida dessas pessoas. Desse modo, houve um aumento significativo de pacientes doadores renais vivos portadores de rim único. Apesar dessas pessoas aparentemente apresentarem uma qualidade de vida semelhante à população geral ${ }^{1}$, há vários estudos indicando haver piora da função renal a longo prazo ${ }^{2-6}$.

Majoritariamente, as doenças que contribuem para o desenvolvimento de Doença Renal Crônica (DRC) compartilham de uma fisiopatologia semelhante baseada na hiperfiltração e hipertensão glomerular 7,8 . 0 principal mecanismo que contribui para o aumento da pressão intraglomerular consiste em meios compensatórios intrarenais que regulam a vasodilatação da arteríola aferente e/ou vasoconstrição da arteríola eferente, via ativação do eixo renina-angiotensina-aldosterona8. Esse mecanismo visa aumentar a Taxa de Filtração Glomerular (TFG) por néfron e, assim, compensar a queda da TFG global. Entretanto, esse aumento de pressão intraglomerular acarreta lesão da sua própria estrutura, dando origem a um processo que leva a esclerose glomerular, diminuindo a quantidade de parênquima renal sadio e, desse modo, reduzindo a TFG que se traduz em aparecimento de proteinúria e progressão para DRC8-10.

Uma das principais doenças que causam o fenômeno de hiperfiltração glomerular é o diabetes mellitus (DM). Em pacientes diabéticos há diminuição da resistência vascular da arteríola aferente por um mecanismo que ainda não é esclarecido, mas a concentração elevada de insulina, IGF-1, peptídeo natriurético atrial e produtos finais da glicosilação de proteínas ocasionam aumento da sinalização intra-renal de óxido nítrico, levando a vasodilatação8 ${ }^{8}$. Assim como ocorre na DM, as dietas ricas em proteínas também podem causar hiperfiltração glomerular pelo mesmo mecanismo de vasodilatação da arteríola aferente ${ }^{7-10}$

Para prevenir tais eventos, a terapia de escolha para pacientes com DRC são os inibidores da enzima conversora da angiotensina (iECA). A principal lógica por trás dessa terapia é o bloqueio do sistema reninaangiotensina-aldosterona, que, por sua vez, inibirá a vasoconstrição da arteríola eferente, diminuindo a pressão intraglomerular e a consequente hiperfiltração ${ }^{7}$.

Em relação aos pacientes uninefrectomizados, ocorre hiperfiltração glomerular compensatória a perda aguda de $50 \%$ da massa renal. Brenner e colaboradores, há mais de três décadas atrás, mostraram, em um estudo experimental com ratos submetidos à nefrectomia, que os néfrons remanescentes apresentavam aumento significativo na filtração e na pressão intraglomerular 12 . Curiosamente, essa hiperfiltração ocorre de maneira muito mais branda em relação a outras doenças renais ${ }^{1,7,11}$. A TFG se altera muito discretamente ao longo do tempo, contudo, há o aparecimento de proteinúria assintomática nesses pacientes. Porém, vale ressaltar que a presença de proteinúria é o sinal mais precoce de lesão glomerular e pode indicar um início de um processo de glomeruloesclerose, sendo mascarado pelos mecanismos compensatórios intra-renais de aumento da TFG, estabilizando a TFG total1-4,6,12,13.

Tendo em vista o exposto, este estudo visa analisar as repercussões clínicas e laboratoriais no longo prazo de pacientes nefrectomizados, analisando a função renal com a prevalência de proteinúria e o cálculo da TFG estimada pela medida longitudinal da creatinina sérica.

\section{MATERIAIS E MÉTODOS}

Estudo retrospectivo, analítico e descritivo, com coleta de dados por meio de análise de prontuários de pacientes portadores de rim único por motivo de nefrectomia. Foram revisados os prontuários dos pacientes com diagnóstico de rim único no ambulatório de nefrologia do HC-UFPR, sendo avaliados dados clínicos como idade, gênero, peso, altura e comorbidades (hipertensão e diabetes mellitus), sedimento urinário em amostra isolada de urina e creatinina sérica.

Os pacientes foram selecionados buscando-os no sistema de informática do HC-UFPR pelos CID-Z52.4 (doador de rim) e CID-Z90.5 (ausência adquirida do rim). Os critérios de inclusão eram pacientes acima dos 18 anos, portadores de rim único e acompanhados, de forma regular, nos ambulatórios de Nefrologia do HC-UFPR no período de 1984 a 2016. 0 único critério de exclusão foi o de preenchimento incompleto de prontuários. Pela metodologia empregada, inicialmente foram encontrados 65 pacientes, destes, 37 não atenderam os critérios da pesquisa: 24 não eram portadores de rim único e 13, embora nefrectomizados, não foram seguidos nos ambulatórios de nefrologia do HC-UFPR. Portanto, 28 pacientes foram incluídos no estudo. Os dados foram coletados por meio de um formulário confeccionado e preenchido pelos pesquisadores (APÊNDICE 1). A função renal foi avaliada por meio da estimativa da TFG, calculada usando a fórmula CKD-EPI (Chronic Kidney Disease Epidemiology collaboration equation) $14 \mathrm{e}$ pelo aparecimento de proteinúria na avaliação de sedimento urinário ao longo do acompanhamento clínico ambulatorial. Para o cálculo da TFG estimada e a verificação da presença de proteinúria foram levados em conta os resultados do primeiro e último exame laboratorial realizados pelos pacientes durante 0 acompanhamento clínico ambulatorial.

0 projeto de pesquisa foi submetido ao Comitê de Ética em Pesquisa em Seres Humanos (CEP) do HC-UFPR e posteriormente aprovado

(CAAE: 


\section{Análise estatística}

Os dados foram analisados quanto à normalidade de sua distribuição. Quando apresentaram distribuição normal, foi realizado o teste t de Student para comparação de grupos. Caso contrário, foi utilizada a comparação por teste de Wilcoxon. 0 teste de Mcnemar foi utilizado para a comparação de dados nominais. A análise de associação foi feita por teste de Fisher. As variáveis foram correlacionadas utilizando teste de correlação de Pearson (dados paramétricos) ou teste de Spearman (dados não paramétricos). Os resultados foram expressos como média (mínimo; máximo) ou média \pm desvio padrão. Valores de $\mathrm{P}$ $<0,05$ foram considerados estatisticamente significativos. Foram utilizados os softwares de análise estatística Prism 7, JMP Statistical Discovery versão 13 e R: A language and environment for statistical computing versão 2.2.5.

\section{RESULTADOS}

A população estudada foi majoritariamente feminina (67\%) e possuía média de idade de $56 \pm 12$ anos. Somente um paciente era afrodescendente. As prevalências de hipertensão arterial sistêmica (HAS) e diabetes mellitus II (DM II) foram de $57 \%$ e $11 \%$, respectivamente. A principal indicação de nefrectomia foi a doação renal (75\%), seguida de litíase renal (21\%). Os pacientes foram acompanhados por uma média de 14,64 \pm 9,02 anos (Tabela 1).

Tabela 1: Características gerais da população

$n=28$

\begin{tabular}{ll}
\hline Gênero (\%) & 33 \\
-Masculino & 67 \\
-Feminino & $56 \pm 12$ \\
Idade (anos) & \\
Etnia (\%) & 96,4 \\
-Brancos & 3,6 \\
-Negros & $14,64 \pm 9,02$ \\
Acompanhamento (anos) & \\
Causa do rim único (\%) & 75 \\
-Doadores & 21,4 \\
-Nefrolitíase & 3,6 \\
-Estenose de JUP & \\
Prevalência de comorbidades (\%) & 11 \\
-DM II & 57 \\
\hline -HAS & \\
\hline \hline & Valores apresentados em percentuais e média \pm desvio padrão \\
\hline
\end{tabular}

A TFG no início do acompanhamento foi de 79,06 $\pm 22,81 \mathrm{ml} / \mathrm{min} / 1,73 \mathrm{~m}^{2}$, ao final do acompanhamento, cujo tempo médio foi de 176,6 meses, e de 70,99 \pm 19,1 $\mathrm{ml} / \mathrm{min} / 1,73 \mathrm{~m}^{2}(P=0,15)$ (Gráfico 1). Inicialmente, $11 \%$ da população estudada apresentou proteinúria e no final, (tempo de acompanhamento: 166,2 \pm 103,7 meses), 39\% dos pacientes $(P<0,05)$ apontaram para a presença de proteína nos exames de urina (Gráfico 2). Ao se analisar mais detalhadamente os pacientes que apresentaram proteinúria na evolução, observou-se que nove pacientes (32\%) desenvolveram ao longo do acompanhamento.

Gráfico 1: Taxa de filtração glomerular no início e no final do acompanhamento

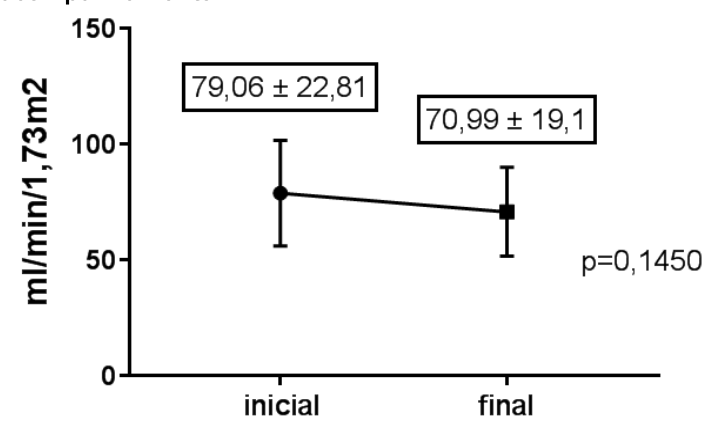

Não se verificou correlação significativa entre TFG final e idade $(r=-0,3016$ e $p=0,11)$. Não se observou diferença significativa entre a presença de DM, HAS, TFG e idade entre os pacientes que apresentavam proteinúria ou não (Tabela 2).

A subamostra de doadores estudada também foi majoritariamente do sexo feminino (62\%). A média da idade foi de $56,8 \pm 12,04$ anos. As prevalências de hipertensão arterial sistêmica e de diabetes mellitus II foram de $52 \%$ e $10 \%$, respectivamente. 0 período médio de acompanhamento foi de $17,33 \pm 8,8$ anos. As médias das TFG inicial e final foram de 79,66 $\pm 21,95$ $\mathrm{ml} / \mathrm{min} / 1,73 \mathrm{~m}^{2}$ e $69,39 \pm 20,52 \mathrm{ml} / \mathrm{min} / 1,73 \mathrm{~m}^{2}(\mathrm{P}=$ $0,14)$ (Gráfico 3), sendo esta verificada após um período médio de 210,3 $\pm 107,1$ meses pós nefrectomia. Quanto à proteinúria, 14\% apresentaram proteinúria inicial e $42 \%$ ( = 0,07) ao final após197,7 \pm 100,3 meses (Gráfico 4).

Gráfico 2: Incidência de proteinúria ao longo do acompanhamento Incidência de proteinúria

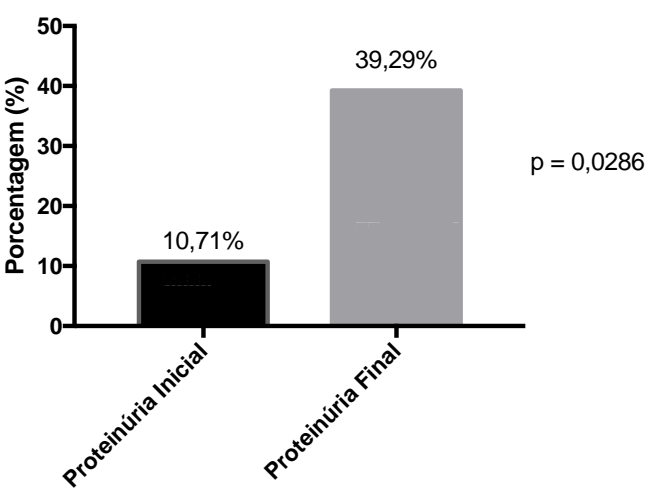


Tabela 2: Comparaçáo de dados clínicos de pacientes com a presença ou ausência de proteinúria

\begin{tabular}{|c|c|c|c|}
\hline & Sam proteinúria & Com proteinúria & Valor $\mathbf{p}$ \\
\hline Gênero (F) (\%) & 57,89 & 42,11 & $p=0,65$ \\
\hline HAS (\%) & 56,25 & 43,75 & $p=0,57$ \\
\hline DM (\%) & 66,67 & 33,33 & $p=0,82$ \\
\hline IMC (kg/m²) & $27,14 \pm 5,79$ & $26,00 \pm 3,59$ & $p=0,67$ \\
\hline $\begin{array}{l}\text { TFG final } \\
\left(\mathrm{ml} / \mathrm{min} / 1,73 \mathrm{~m}^{2}\right)\end{array}$ & $76,41 \pm 19,56$ & $62,61 \pm 15,94$ & $p=0,12$ \\
\hline Idade (anos) & $56,64 \pm 14,43$ & $55,36 \pm 7,72$ & $p=0,74$ \\
\hline
\end{tabular}

Gráfico 3: Evolução da taxa de filtração glomerular em doadores

Evolução da TFG em doadores

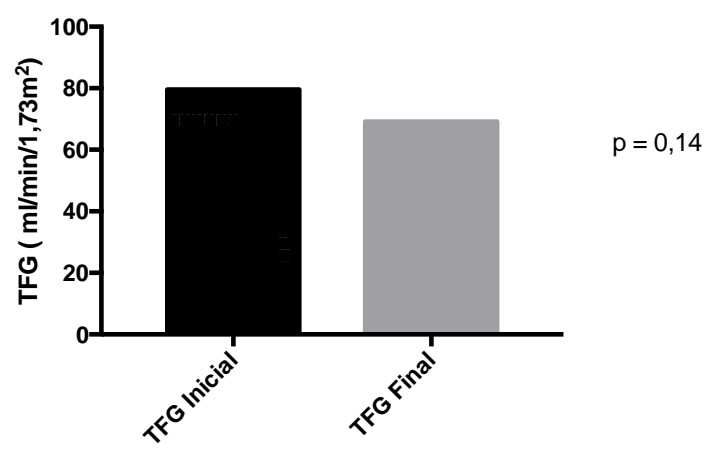

Gráfico 4: Incidência de proteinúria nos doadores ao longo do acompanhamento

\section{Proteinúria nos doadores}

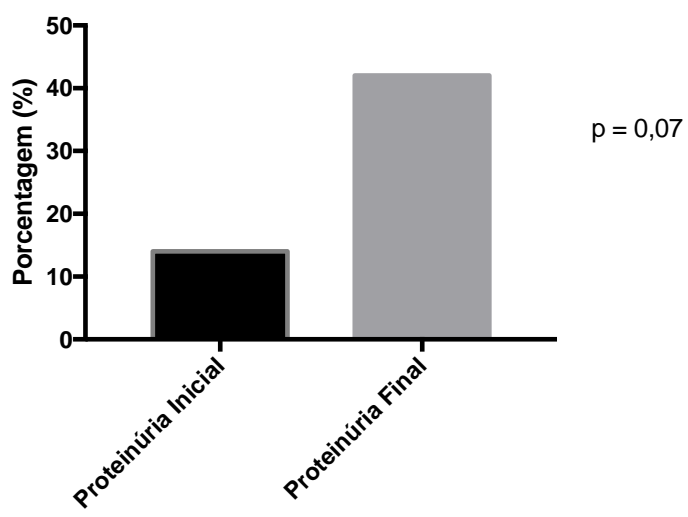

\section{DISCUSSÃO}

Os principais achados do presente estudo apontam que a população estudada era majoritariamente do sexo feminino, sendo que a principal causa de nefrectomia foi a doação renal e com uma média de acompanhamento ao longo de 14,64 $\pm 9,02$ anos. No presente estudo, foi observado um aumento significativo da prevalência de proteinúria o que leva a crer que a presença de rim único pode ser um fator para o desenvolvimento de DRC. Semelhantes aos nossos achados, alguns estudos correlacionaram a doação renal com o surgimento de proteinúria ${ }^{2,15,16}$, sendo o sexo masculino identificado como um fator de risco adicional1,2,16. No presente estudo, não conseguimos mostrar associação entre proteinúria e sexo masculino, provavelmente pela composição majoritariamente feminina da população estudada. Nos estudos que acompanharam crianças nefrectomizados por neoplasias renais, a proteinúria também foi uma das anormalidades identificadas ${ }^{17-19}$.

Em um estudo onde a função renal de mais de 3500 doadores renais foi avaliada, Ibrahim et al. demonstraram que a função renal não sofre prejuízo, embora haja desenvolvimento de proteinúria que apresenta correlação direta com o tempo desde a doação1. Outros estudos também minimizam a perda da função renal em doadores em termos de queda do da TFG2,16,20. No presente estudo, se verificou uma tendência ao aumento da incidência de proteinúria $(p=0,07)$ ao se analisar somente os doadores renais.

0 aparecimento da proteinúria pode ser um fator preditor da disfunção renal mais fiel que a diminuição da TFG por causa do papel atenuante da hiperfiltração. Em virtude da hiperfiltração nos glomérulos remanescentes e do mecanismo de aumento da secreção tubular da creatinina (que compensaria a menor excreção glomerular), o aumento da creatinina sérica pode ser considerado um sinal tardio de perda de função renal, o que poderia explicar a não redução na TFGe observada no presente estudo. A hiperfiltração é responsável pela manutenção da função renal após a nefrectomia unilateral em doadores. Mesmo após duas décadas, a TFG total permanece aproximadamente $70 \%$ dos valores prénefrectomia mesmo com a perda de $50 \%$ da massa renal ${ }^{7}$. 
Nos modelos animais onde a hiperfiltração se mostrou deletéria e causou perda significativa da função renal, esclerose glomerular, proteinúria e hipertensão, houve retirada de $5 / \%$ da massa renal 21 . Em seres humanos submetidos a nefrectomia subtotal também foram observados alterações histológicas semelhantes 22,23 . De modo similar, a incidência de insuficiência renal foi muito maior em pacientes nefrectomizados por consequência de tumor de Wilms bilateral quando comparados àqueles portadores do tumor de Wilms unilateral24. Uma vez que todos os nossos pacientes foram submetidos a apenas nefrectomia unilateral, a preservação da função renal em termos de TFG está de acordo com a literatura e se deve à hiperfiltração.

Gonzales et al. evidenciaram um aumento da hiperfiltração, secundário à obesidade, e o seu efeito deletério a longo prazo sobre a função renal em pacientes com perda de massa renal e esclerose glomerular focal 25 . De modo análogo, a nefropatia diabética, a maior causa de doença renal crônica no mundo, pode ser um grande agravante para os pacientes com rim único. É provável que a hiperfiltração da nefropatia diabética, secundária a uma desregulação hemodinâmica ${ }^{26}$, antecipe ou resulte em lesões glomerulares esclerosantes focais e insuficiência renal. No nosso estudo, havia somente 3 participantes diabéticos, o que impossibilitou a associação da diabetes com marcos de dano renal.

\section{CONCLUSÃO}

Nesta série de casos, pode-se verificar que pacientes nefrectomizados podem desenvolver proteinúria ao longo do tempo apesar de não se observar uma queda significativa da TFGe, apontando para a possibilidade que estes indivíduos desenvolvam DRC. Isto faz com que pacientes que sejam portadores de rim único necessitem de acompanhamento ambulatorial nefrológico contínuo visando o diagnóstico de disfunção renal precoce (microalbuminúria) e de outros reconhecidos fatores de risco para o desenvolvimento de DRC como DM e a HAS. Além disso, reforça-se a necessidade de seleção criteriosa de potenciais doadores renais para que estes não se tornem portadores de DRC no longo prazo.

\section{REFERÊNCIAS}

1. Ibrahim HN, Foley R, Tan L, Rogers T, Bailey RF, Guo $\mathrm{H}$, et al. Long-term consequences of kidney donation. N Engl J Med. 2009; 360: 459-469.

2. Goldfarb DA, Matin SF, Braun WE, Schreiber MJ, Mastroianni B, Papajcik D, et al. Renal outcome 25 years after donor nephrectomy. Journal of Urology. 2001; 166(6): 2043-2047.

3. Basturk T, Koc Y, Ucar Z, Sakaci T, Ahbap E, Kara E, et al. Renal Damage Frequency in Patients with Solitary Kidney and Factors That Affect Progression. Int J Nephrol. Vol. 2015; ID 876907, 7 pgs.
4. Argueso LR, Ritchey ML, Boyle ET, Milliner DS, Bergstralh EJ, Kramer SA. Prognosis of children with solitary kidney after unilateral nephrectomy. Journal of Urology. 1992; 148(2): 747-751.

5. de Lucas C, Nocea A, San Roman J, Espinola B, Ecija JL, Martul MV. Solitary kidney. Study of renal morphology and function in 95 children. Nefrologia. 2006; 26(1): 56-63.

6. Westland R, Schreuder MF, van Goudoever JB, Sanna-Cherchi S, van Wijk JA. Clinical implications of the solitary functioning kidney. Clin J Am Soc Nephrol. 2014;9(5):978-86.

7. Brenner BM, Lawler EV, Mackenzie HS. The hyperfiltration theory: A paradigm shift in nephrology. Kidney International. 1996; 49(6): 1774-1777.

8. Helal I, Fick-Brosnahan GM, Reed-Gitomer B, Schrier RW. Glomerular hyperfiltration: definitions, mechanisms and clinical implications. Nat Rev Nephrol. 2012; 8(5): 293-300.

9. Krill A, Salami S, Rosen L, Friedman SC, Gitlin J, Palmer LS. Evaluating Compensatory Hypertrophy: A Growth Curve Specific for Solitary Functioning Kidneys. Journal of Urology. 2012; 188(4): 16131617.

10. Neuringer JR, Brenner BM. Glomerular hypertension cause and consequence of renal injury. Journal of Hypertension. 1992; 10: S91-S97.

11. Blantz RC, Steiner RW. Benign hyperfiltration after living kidney donation. J Clin Invest. 2015; 125(3): 972-974.

12. Hostetter TH, Olson JL, Rennke HG, Venkatachalam MA, Brenner BM. Hyperfiltration in remnant nephrons: a potentially adverse response to renal ablation. Am J Physiol. 1981; 241(1): F85-93.

13. Shapiro E, Goldfarb DA, Ritchey ML. The congenital and acquired solitary kidney. Rev Urol. 2003; 5(1): 2 8.

14. Levey AS, Stevens LA, Schmid CH, et. al. for the CKDEPI (Chronic Kidney Disease Epidemiology Collaboration). A New Equation to Estimate Glomerular Filtration Rate. Ann Intern Med. 2009 May 5; 150:604-612.

15. Bock HA, Bachofen M, Landmann J, Thiel G. Glomerular hyperfiltration after unilateral nephrectomy in living kidney donors. Transplant International Official Journal of the European Society for Organ Transplantation. 1992;:156-9

16. Hakim RM, Goldszer RC, Brenner BM. Hypertension and proteinuria: Long-term sequelae of uninephrectomy in humans. Kidney International. 1984;25(6):930-6.

17. Levitt GA, Yeomans E, Dicks Mireaux C, et al. Renal size and function after cure of Wilms' tumor. $\mathrm{Br} J$ Cancer. 1992;66:877-882.

18. Baudoin P, Provoost AP, Molenaar JC. Renal function up to 50 years after unilateral nephrectomy in childhood. Am J Kidney Dis. 1993;21:603-611.

19. Robitaille P, Mongeau JG, Lortie L, Sinnassamy P. Long-term follow-up of patients who under- went nephrectomy in childhood. Lancet. 1985;1:12971299. 
20. Najarian J. 20 years or more of follow-up of living kidney donors. The Lancet. 1992;340(8823):80710.

21. Shimamura $\mathrm{T}$, Morrison AB. A progressive glomerulosclerosis occurring in partial five- sixths nephrectomized rats. Am J Pathol. 1975;79:95-101

22. Novick AC, Gephardt G, Guz B, Steinmuller D, Tubbs RR. Long-Term Follow-up after Partial Removal of a Solitary Kidney. New England Journal of Medicine. 19910ct;325(15):1058-62

23. Praga M, Morales E, Herrero JC, Campos AP, Domínguez-Gil B, Alegre $\mathrm{R}$, et al. Absence of hypoalbuminemia despite massive proteinuria in focal segmental glomerulosclerosis secondary to hyperfiltration. American Journal of Kidney Diseases. 1999;33(1):52-8.

24. Ritchey ML, Green DM, Thomas P, et al. Renal failure in Wilms' tumor patients: a report from the National Wilms' Tumor Study Group. Med Pediatr Oncol. 1996;26:75-80

25. Gonzalez E, Gutierrez E, Morales E, Hernandez E, Andres A, Bello I, et al. Factors influencing the progression of renal damage in patients with unilateral renal agenesis and remnant kidney. Kidney International. 2005;68(1):263-70.

26. O'Bryan GT, Hostetter TH. The renal hemodynamic basis of diabetic nephropathy. Semin. Nephrol. 1997 Mar;17(2):93-100. 